\title{
Centrifuge experiments for the determination of the moisture storage and transport properties in the overhygroscopic range
}

\author{
Evy Vereecken ${ }^{1, *}$, Staf Roels ${ }^{1}$, and Hans Janssen ${ }^{1}$ \\ ${ }^{1}$ KU Leuven, Building Physics Section, Kasteelpark Arenberg 40, 3001 Leuven, Belgium
}

\begin{abstract}
This study explores the potential of a centrifuge experiment for hygric property determination. In this method, a core sample is placed in a tube which is spun at different rotational speeds to create a distribution of moisture contents and capillary pressures. This way, a simultaneous determination of the moisture retention curve and moisture permeability curve is possible. Measurements performed in the petroleum industry and in soil science already showed the technique to be an appropriate substitute for the pressure plate method. In building physics, the potential of the centrifuge method is currently still unexplored. Therefore, in this study, preliminary desorption measurements on brick samples are performed. To infer the moisture retention curve, an approximate data analysis method is applied. This approach is compared to a more sophisticated parameter estimation technique, which is also used to infer the moisture permeability curve. From a theoretical point of view, the centrifuge method allows a simultaneous determination of the moisture storage and transport properties of building materials. In practice, however, experimental inaccuracies make the experimental results unusable for a reliable determination of the moisture transport properties.
\end{abstract}

\section{Introduction}

Moisture is often a key factor in damage or discomfort in buildings, and the correct analysis and design of buildings hence requires a reliable assessment of moisture transfer in building materials. Such assessment necessitates knowledge on the moisture storage and transport properties of porous materials. Traditionally, the porous plate method or Mercury Intrusion Porosimetry (MIP) are applied to characterise the materials' moisture storage properties in the overhygroscopic range. Unfortunately, the pressure plate method needs weeks till months of experiments to obtain the moisture retention curve, while MIP is an anything but eco-friendly technique. Similarly, to measure the moisture transport properties timeconsuming and often labour-intensive tests are needed (e.g.[1]).

This paper explores the potential of a centrifuge method to measure both the moisture retention curve and the moisture permeability curve in the overhygroscopic range and this within an acceptable time span. Thereto, in the centrifuge method, the test samples are spun

*Corresponding author: Evy.Vereecken@kuleuven.be 
at a series of different rotational speeds, creating a distribution of moisture contents and capillary pressures over the test sample. Measurements performed in soil science already showed the technique to be an appropriate substitute for the characterization of the moisture storage properties [2], and, moreover, proved the added value of the dynamic behaviour for the determination of the moisture transport properties [3]. In building physics, the potential of the centrifuge method is currently still unexplored. Therefore, in this study, exploratory desorption measurements by use of a ultracentrifuge are performed on a typical building material. To obtain the moisture retention curve from the experimental data, an approximate data analysis method as proposed in the petroleum industry is applied. Next, this approach is compared to a more sophisticated parameter estimation technique. Ultimately, the transient behaviour is used to infer the permeability curve.

\section{The centrifuge experiment}

\subsection{Main principle}

In the centrifuge method, a core sample is placed in a tube which is spun at different rotational speeds to create a distribution of moisture contents and capillary pressures. The one-dimensional moisture flow in the sample can be expressed by the modified version of the Richards equation for a centrifugal field:

$$
\frac{\partial w}{\partial t}=\frac{\partial}{\partial r}\left[K \cdot\left(\frac{\partial p_{c}}{\partial r}-\rho \omega^{2} r\right)\right]
$$

with $w$ the moisture content $\left(\mathrm{kg} / \mathrm{m}^{3}\right), t$ the time (s), $K$ the permeability (s), $p_{c}$ the capillary pressure $(\mathrm{Pa}), \rho$ the liquid density $\left(\mathrm{kg} / \mathrm{m}^{3}\right), \omega$ the rotational speed $(\mathrm{rad} / \mathrm{s})$ and $r$ the distance from the centre of rotation $(\mathrm{m})$. By sequentially increasing the rotational speed, the moisture in the sample will flow to the sample's outlet surface and will finally be forced out of the core. A drainage of the sample will be obtained. In equilibrium, the capillary pressure at a distance $r$ from the centre of rotation $p_{c}(r)(\mathrm{Pa})$ is given by (Figure 1a):

$$
p_{c}(r)=\frac{\Delta \rho \omega^{2}}{2} \cdot\left(r_{2}^{2}-r^{2}\right)
$$

with $\Delta \rho$ the difference in density between the invading fluid (air) and the displaced fluid (water), $r_{2}$ the distance of the sample's outlet face from the centre of rotation $(\mathrm{m})$ and $r$ the distance of the point concerned from the centre of rotation $(\mathrm{m})$. An example of the capillary pressure variation over a test sample is shown in Figure $1 \mathrm{~b}$.

\subsection{Experiment}

The centrifuge measurements are performed by use of a Beckman ultracentrifuge (Figure 2) equipped with a PIR-122 rotor. Three ceramic brick samples with a diameter of $2.47 \mathrm{~cm}$, a length of $3.99 \mathrm{~cm}$ and a porosity between $32.2 \%$ and $33.4 \%$ were measured simultaneously in one test run. The radius between the centre of rotation and the sample's top face $\left(r_{l}\right)$ was $5.39 \mathrm{~cm}$. Traditionally, the centrifuge measurement starts from a fully saturated sample. In the current study, however, only two samples were vacuum saturated before the start of the centrifuge measurement; the third sample was capillary saturated. The rotational speeds given in Table 1 were imposed to the samples. The expelled water was collected in a 
receiving tube, and the volume of expelled water was registered by converting pixel readings taken from images that were automatically captured by use of a camera unit.

a)

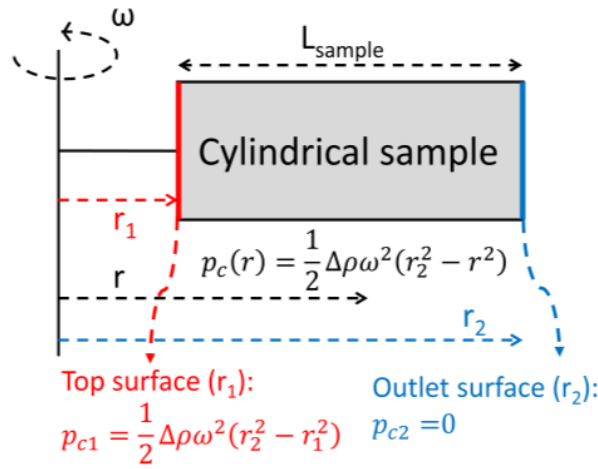

b)

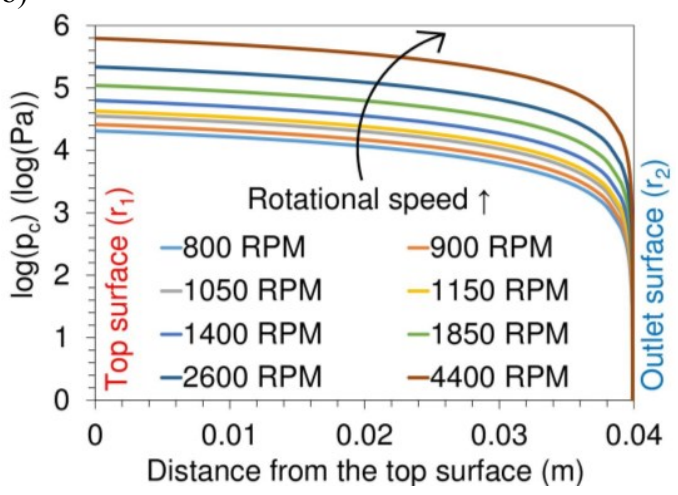

Fig. 1. Spatial capillary pressure at equilibrium in the centrifuge experiment: (a) schematic explanation of the parameters in Eq.(2), (b) capillary pressure as a function of location for a series of rotational speeds $\left(L_{\text {sample }}=3.99 \mathrm{~cm}, r_{l}=5.39 \mathrm{~cm}\right)$.

a)

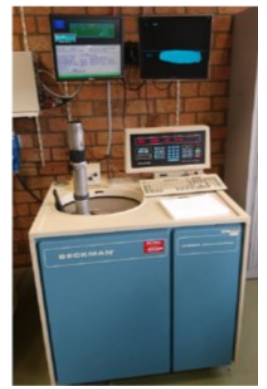

b)

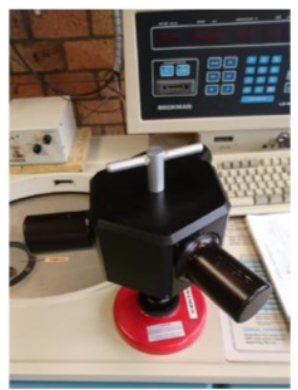

c)

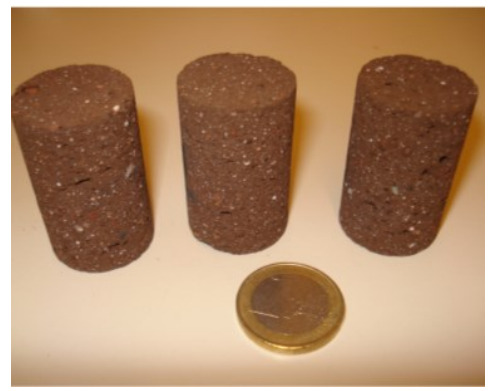

Fig. 2. Test setup: (a) ultracentrifuge, (b) rotor with a holder for the test samples, (c) brick samples.

Table 1. Imposed rotational speed together with the corresponding capillary pressure at the sample's top $\left(r_{l}\right.$ in Figure 1a).

\begin{tabular}{|c|c|c|c|c|c|c|c|c|}
\hline Start time (h) & 0 & 24 & 48 & 72 & 95.5 & 119.5 & 143.5 & 163.5 \\
\hline Rotational speed (RPM) & 800 & 900 & 1050 & 1150 & 1400 & 1850 & 2600 & 4400 \\
\hline $\log \left(\mathbf{p}_{\mathbf{c}}\right)(\log (\mathbf{P a}))$ & 4.25 & 4.40 & 4.50 & 4.60 & 4.80 & 5.05 & 5.35 & 5.80 \\
\hline
\end{tabular}

\subsection{Experimental output}

Figure 3a shows the expelled fluid volume as a function of experimental time. At low rotational speed, highly scattered readings were obtained. Furthermore, a distinct difference in the fluid volume expelled from Sample 1 and Sample 2 (both initially vacuum saturated) can be observed. After each change in rotational speed, a new equilibrium in expelled fluid volume was obtained in less than 30 minutes. Though, the measurement accuracy was found to be inadequate to register smooth curves for the transient behaviour (Figure 3b). 
a)

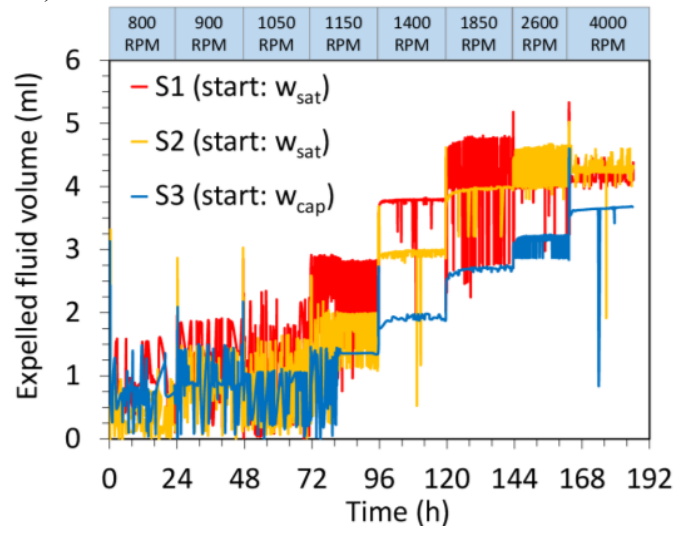

b)

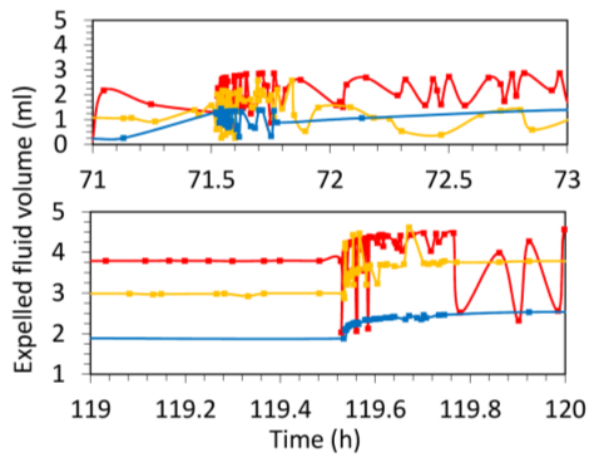

Fig. 3. Expelled fluid volume as a function of time together with the imposed rotational speed: (a) total run, (b) detailed view in the intervals $71-73 \mathrm{~h}$ and $119-120 \mathrm{~h}$.

\section{Data-processing}

The centrifuge method determines the hygric material properties in an indirect way, as, during the measurement, the capillary pressure and thus the moisture properties vary over the length of the test sample. Approximate equilibrium methods or inverse modelling techniques are needed to convert the sample's average moisture content into the correct link between the moisture potential and the material's moisture storage and transport properties.

\subsection{Equilibrium analysis}

At hydrostatic equilibrium, the average moisture content $\bar{w}\left(\mathrm{~kg} / \mathrm{m}^{3}\right)$ of the core is given by:

$$
\bar{w}=\frac{1}{r_{2}-r_{1}} \int_{r_{1}}^{r_{2}} w\left(p_{c}(\omega, r)\right) d r
$$

with $w\left(p_{c}(\omega, r)\right)$ the moisture content $\left(\mathrm{kg} / \mathrm{m}^{3}\right)$ at a distance $r$ from the centre of rotation when a rotational speed $\omega$ is imposed, and $r_{1}$ and $r_{2}(\mathrm{~m})$ the distance of respectively the outlet and the top surface to the centre of rotation (Figure 1a). Several procedures to solve Eq.(3) are applied in the petroleum and soil industry [4]. In the current study, first, Forbes Second method (see [4]) was applied to convert the average moisture content (which can be calculated based on the expelled fluid volume) into the moisture content at the sample's top surface ( $r_{l}$ in Figure 1a). The capillary pressure at this top surface can be determined by Eq.(2), and thus, in this way the moisture retention curve can be determined. The results are shown in Figure 4. For the initially vacuum saturated samples (Figure 4a), the Forbes Second method yield an unrealistically high moisture content in the capillary pressure range above $5 \log (\mathrm{Pa})$. In the higher moisture range, the results obtained via the Forbes Second method are in close agreement with the MIP results. For the initially capillary saturated sample (Figure 4b), a good agreement between centrifuge measurements and MIP was achieved when using Forbes Second method.

Next, another data-processing method was applied. A moisture storage function was fitted by minimizing the difference between the measured and simulated expelled fluid volumes at equilibrium. As a parametric function, a van Genuchten model was assumed: 


$$
w=w_{\max }\left[1+\left(\alpha \cdot p_{c}\right)^{n}\right]^{(1 / n)-1}
$$

with $w$ the moisture content $\left(\mathrm{kg} / \mathrm{m}^{3}\right), p_{c}$ the capillary pressure $(\mathrm{Pa}), w_{\max }$ the vacuum (for Sample 1 and 2) or capillary saturated (for Sample 3) moisture content $\left(\mathrm{kg} / \mathrm{m}^{3}\right)$ and $\alpha$ and $n$ fitting parameters. The expelled fluid volumes at hydrostatic equilibrium were simulated by use of a control volume method. As shown in Figure 4, by use of this approach also for Sample 1 and 2 a good agreement was achieved for the range above $5 \log (\mathrm{Pa})$. Remark furthermore the good agreement between the moisture retention curves for Sample 1 and 2, and this despite the difference in expelled fluid volume (Figure 3a).

a)

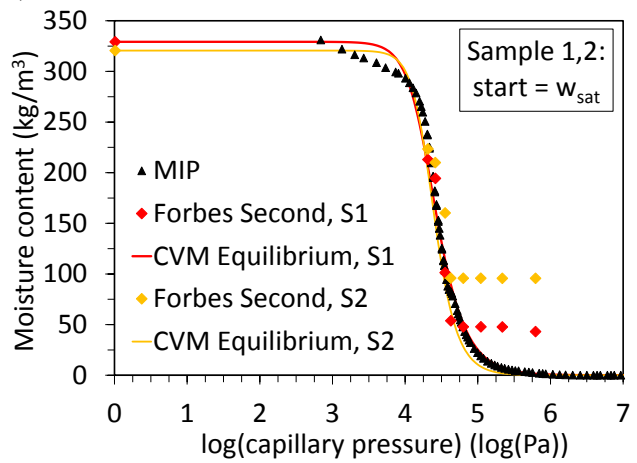

b)

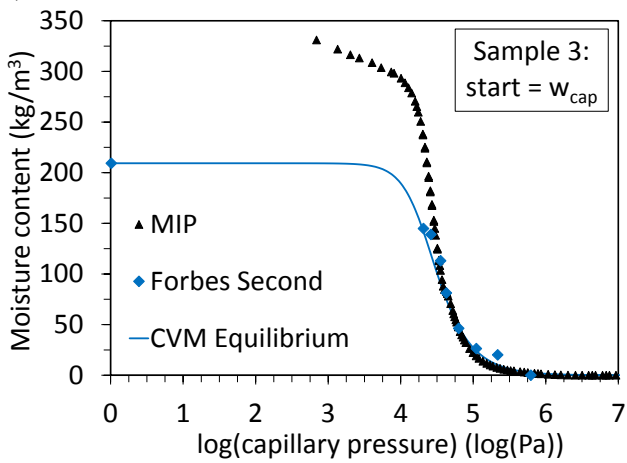

Fig. 4. Moisture retention curve achieved based on the drainage centrifuge measurements started from (a) vacuum saturation, (b) capillary saturation. As a comparison the results of a MIP measurement (starting from $\mathrm{w}_{\text {sat }}$ ) are shown.

\subsection{Dynamic analysis}

The dynamic behaviour of the centrifuge measurement could be used to infer the moisture transport properties. In the current study, the centrifuge measurement is simulated by use of a modified version of HYDRUS-1D [3] that solves Eq.(1) via finite element schemes. At the sample's top surface $\left(r_{1}\right)$ a constant zero flux was imposed. At the outlet surface $\left(r_{2}\right)$ time variable boundary conditions given by the rotational speeds in Table 1 were imposed. The moisture retention curve was set to the van Genuchten function determined in Section 3.1. The initial moisture content of the sample was set at the vacuum saturated (for Sample 1,2 ) or capillary saturated moisture content (for Sample 3 ). The permeability $K$ (s) was modelled by a van Genuchten-Mualem permeability function:

$$
K=K_{\max } S^{l}\left[1-\left(1-S^{1 / m}\right)^{m}\right]^{2}
$$

with the saturation degree $S(-)$ and the parameter $m(=1-1 / \mathrm{n})$ linked to the moisture retention curve. The parameter $K_{\max }(\mathrm{s})$ represents the vacuum (Sample 1,2) or capillary (Sample 3) saturated permeability and is together with the parameter $l$ inversely modelled. Thereto the difference between the numerical and the experimental times series of the expelled moisture volumes is minimized via the Levenberg-Marquardt algorithm (see Figure $5 \mathrm{a}$ for an example). Figure $5 \mathrm{~b}$ shows the permeability as obtained for the three test samples. The estimated curves deviate from the curve obtained via the X-ray projection technique [1] and from each other. The scattered experimental output that avoids a clear 
interpretation of the transient behaviour (Figure 3b) lies at the base of the unreliable permeability curves. For noise-free data, it should be possible to infer the permeability. Indeed, when creating artificial data for a similar centrifuge experiment (see [5] for the principle applied on moisture transport in the hygroscopic range) on a brick sample with known moisture storage and transport properties, both the moisture retention and the permeability curve could be accurately inferred (not shown).

a)

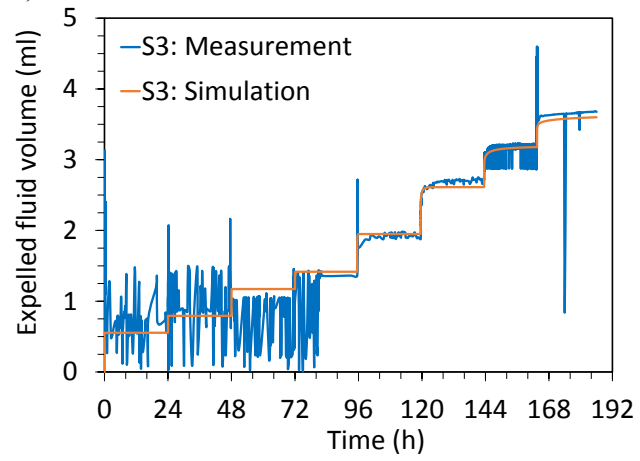

b)

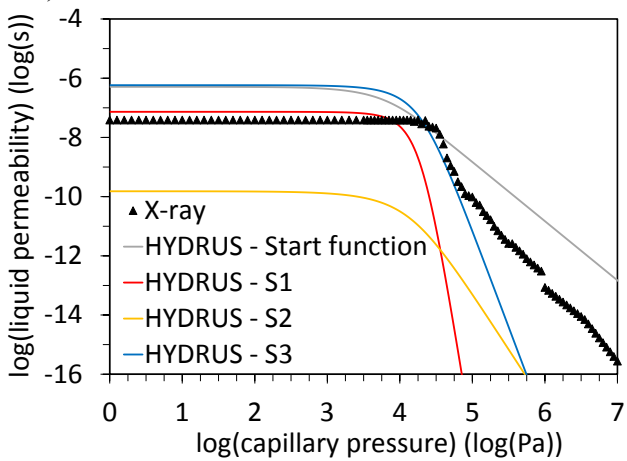

Fig. 5. (a) Comparison between experimentally measured and numerically simulated expelled fluid volume, (b) Permeability curves obtained via inverse modelling with HYDRUS-1D for the three samples together with the results obtained via the X-ray projection method. For completeness, also the starting permeability curve in the Levenberg-Marquardt algorithm is shown.

\section{Conclusions}

The applicability of a centrifuge method to measure the moisture storage and moisture transport properties of building materials has been investigated. The moisture retention curve achieved via inversely fitting a parametric function was in good agreement with the results obtained via MIP. On the other hand, when applying the Forbes Second method, an approximate data analysis method often applied to process centrifuge measurements, an unrealistic moisture retention curve was obtained for the initially vacuum saturated samples. The transient behaviour could, from a theoretical point of view, be used to infer the moisture transport properties. However, in practice, experimental inaccuracies (a.o. scattered readings for the expelled fluid volume) made the measurement data unusable for a reliable determination of the moisture transport properties.

Evy Vereecken is a postdoctoral fellow of the Research Foundation (FWO) - Flanders, Belgium (FWO project $12 \mathrm{~J} 5219 \mathrm{~N}$ ). The measurements within this paper have been financed by the FWO Research Grant $1517817 \mathrm{~N}$. These financial supports are gratefully acknowledged. The centrifuge measurements were performed by PanTerra Geoconsultants, the Netherlands.

\section{References}

1. J. Carmeliet, H. Hens, S. Roels, et al., J Thermal Env Bldg Sci 27(4), 277-305 (2014).

2. A. Reatto, E. da Silva, A. Bruand, et al., Soil Sci Soc Am J 76(6), 1547-1553 (2008).

3. J. Šimůnek, J.R. Nimmo, Water Resour Res, 41(4), W04015, 1-9 (2005).

4. P. Forbes, International Symposium-Society of core analysts, SCA-9714 (Dallas, 1997)

5. E. Vereecken, S. Roels, H. Janssen, Build Environ, 131, 184-196 (2018). 